\title{
Insights into Hydrogen Evolution Reaction on 2D Transition
}

\section{Metal Dichalcogenides}

\author{
Zhenbin Wang, ${ }^{1}$ Michael T. Tang, ${ }^{2,3}$ Ang Cao, ${ }^{1}$ Karen Chan, ${ }^{1}$ and Jens K. Nørskov ${ }^{1, *}$ \\ ${ }^{1}$ Department of Physics, Technical University of Denmark, 2800 Kongens Lyngby, Denmark \\ ${ }^{2}$ Department of Material Science and Engineering, Stanford University, Stanford, California \\ 94305, United States \\ ${ }^{3}$ SUNCAT Center for Interface Science and Catalysis, SLAC National Accelerator Laboratory, \\ Menlo Park, California 94025, United States \\ E-mail: jkno@dtu.dk
}

\begin{abstract}
Understanding the hydrogen evolution reaction (HER) behaviors over 2D transition metal dichalcogenides (2D-TMDs) is critical for the development of non-precious HER electrocatalysts with better activity. In this work, by combining density functional theory calculations with microkinetic modelling, we thoroughly investigated the HER mechanism on 2DTMDs. We find there is an important dependence of simulated cell size on the calculated hydrogen adsorption energy and the activation barrier for $\mathrm{MoS}_{2}$. Distinct from previous "H migration" mechanisms proposed for the Heyrovsky reaction - the rate-determining step for $\mathrm{MoS}_{2}$, we propose the Mo site only serves as the stabilized transition state rather than $\mathrm{H}$ adsorption. In comparison to transition metal electrocatalysts, we find that the activation barrier of the Heyrovsky reaction on 2D-TMDs scales with the hydrogen adsorption energy exactly as for transition metals except that all activation energies are displaced upwards by $c a .0 .4 \mathrm{eV}$. This higher Heyrovsky
\end{abstract}


activation barrier is responsible for the substantially lower activity of 2D-TMDs. We further show that this higher activation barrier stems from the more positively charged adsorbed hydrogen on the chalcogenides interacting repulsively with the incoming proton. Based on these insights, we discuss potential strategies for the design of non-precious HER catalysts with activity comparable to Pt.

Keywords: hydrogen evolution reaction, transition metal dichalcogenides, density functional theory, microkinetic modelling, electrochemical barrier, kinetic activity volcano

\section{Introduction}

Developing earth-abundant and low-cost catalysts toward hydrogen evolution is essential for the implementation of hydrogen-based sustainable energy technologies on a large scale. Twodimensional transition metal dichalcogenides (2D-TMDs) have received considerable interest as promising non-precious electrocatalysts for hydrogen evolution reaction (HER) in acidic media. ${ }^{1-}$

${ }^{3}$ Based on density functional theory (DFT) calculations and using the fact that the hydrogen adsorption free energy $\left(\Delta G_{\mathrm{H}}\right)$ is a good descriptor of HER activity for transition metals, ${ }^{4-11}$ Hinnemann et al. ${ }^{12}$ first proposed that the Mo edge site of $\mathrm{MoS}_{2}$ would be active for HER, which was later extensively verified in experiments. ${ }^{13-19}$ A large number of works have since then explored not only $\mathrm{MoS}_{2}$ but also other 2D-TMDs, phosphides, carbides as HER catalysts. ${ }^{20-27}$ 
$\Delta G_{\mathrm{H}}$ as an HER descriptor was developed based on the HER activity volcano of metals and later widely used for other materials. Non-transition-metal HER catalysts seem to follow the same trend as transition metals, where activity $v s . \Delta G_{\mathrm{H}}$ reaches a maximum at around $\Delta G_{\mathrm{H}}=0 .{ }^{13,28}$ Leveraging DFT calculated $\Delta G_{\mathrm{H}}$, many 2D-TMDs materials have been explored to search for active HER catalysts. ${ }^{29-32}$ All theoretical studies of the HER activity in terms of $\Delta G_{\mathrm{H}}$ were grounded on thermodynamics, and no kinetics has been considered. Recently, the lack of kinetic consideration has raised concerns about whether $\Delta G_{\mathrm{H}}$ is a good HER descriptor or not in the literature. ${ }^{33-38}$ The inclusion of kinetics in the HER activity evaluation asks for calculations of the electrochemical barrier for the rate-determining step, which is a non-trivial task in computation. This is because simulations based on periodic DFT calculations are performed at a constant charge, while the electrochemical reactions in real systems take place at a constant potential. ${ }^{39-41}$ To the best of our knowledge, only a very few studies have been devoted to the HER electrochemical barrier calculations of 2D-TMDs, primarily for $\mathrm{MoS}_{2}$, and the detailed reaction mechanism remains debated. ${ }^{38,42-45}$

On the other hand, extensive research in experiment has been performed to improve the HER activity of 2D-TMDs, ${ }^{1-3,13-25}$ but they commonly exhibit an activity of about three orders of magnitude lower than that of transition metals electrocatalysts. ${ }^{73-15,46-48}$ For example, the measured exchange current density $\left(j_{0}\right)$ of $\mathrm{MoS}_{2}$ in experiment is $1.2 \times 10^{-3} \mathrm{~mA} / \mathrm{cm}^{2} \sim 7.9 \times 10^{-3}$ $\mathrm{mA} / \mathrm{cm}^{2},{ }^{13-15}$ while the experimental $j_{0}$ of $\mathrm{Pt}$ is $0.21 \sim 1.35 \mathrm{~mA} / \mathrm{cm}^{2}{ }^{7,46,48}$ This remarkable difference in activity poses two fundamental questions i) why is $\Delta G_{\mathrm{H}}$ a good descriptor of the catalytic activity for 2D-TMDs, whereas they have the lower rates? ii) whether $\Delta G_{\mathrm{H}} \sim 0$ is still a good design criterion? 
In this work, we address these critical questions by investigating the thermodynamics and kinetics of HER over 2D-TMDs in comparison to transition metal electrocatalysts. We show that the activation energies of the rate-determining step on 2D-TMDs scale with $\Delta G_{\mathrm{H}}$ exactly as for the transition metals electrocatalysts but with $\sim 0.4 \mathrm{eV}$ higher activation energies. Microkinetic modelling further shows that these higher activation energies result in activity volcanoes of 2DTMDs for HER that are similar to those of the transition metals but shifted down in rate by several orders of magnitude. We discuss the origin of the higher activation energy and suggest possible ways to design non-precious HER catalysts with activity approaching Pt.

\section{Results}

Revisiting hydrogen adsorption free energy $\left(\Delta \boldsymbol{G}_{\mathrm{H}}\right)$. $\Delta G_{\mathrm{H}}$ has been widely used to evaluate the HER activity of $\mathrm{MoS}_{2}$, whereas its DFT calculated value in the literature varies from $-0.30 \mathrm{eV}$ to $0.25 \mathrm{eV} .{ }^{12,29,32,38,45,49,50}$ Here we revisited the calculated $\Delta G_{\mathrm{H}}$ on the $\mathrm{MoS}_{2}$ Mo-edge with different cell sizes. Figure 1a shows a representative stripe model of $\mathrm{MoS}_{2}$ with a cell size of $8 \times 4$, where possible $\mathrm{H}$ adsorption sites on the Mo-edge were marked. Based on $\Delta G_{\mathrm{H}} \sim 0$, it is generally believed that $\mathrm{S}$ is the active site for HER, while the kinetic study by Huang et al. ${ }^{38}$ suggested it is the Mo site that determines the HER activity. We therefore also calculated $\Delta G_{\mathrm{H}}$ for the Mo site for comparison. Figure $1 \mathrm{~b}$ presents calculated $\Delta G_{\mathrm{H}}$ at the $\mathrm{S}$ and Mo sites for different simulated cell sizes. We started off $\Delta G_{\mathrm{H}}$ calculations at the S site with the $\mathrm{H}$ coverage $\left(\theta_{\mathrm{H}}\right)$ of $0.5 \mathrm{ML}$, which was reported to be the most energetically stable under HER conditions. ${ }^{29}$ We find that $\Delta G_{\mathrm{H}}$ converges to $0.35 \mathrm{eV}$ from the cell size of $4 \times 4$ to $12 \times 8$, implying that $\theta_{\mathrm{H}}$ of $0.5 \mathrm{ML}$ at the $\mathrm{S}$ site is somewhat high. $\Delta G_{\mathrm{H}}$ with $\theta_{\mathrm{H}}$ of $(0.5 x-1) / x$ ML (a hydrogen removed from $0.5 \mathrm{ML}$ ) was then calculated and converged to $0.03 \mathrm{eV}$ in a $12 \times 8$ model. According to the definition of differential $\Delta G_{\mathrm{H}}$, (See 
Methods) its value of an active site is expected to be converged at around zero. $\theta_{\mathrm{H}}$ now becomes $0.42 \mathrm{ML}$, which lies between the most stable hydrogen coverage of $0.33 \mathrm{ML}$ and $0.5 \mathrm{ML}$ under HER conditions determined using a $3 \times 3$ model $^{50}$ and a $4 \times 4$ model $^{29}$, respectively. As shown in Figure $1 \mathrm{~b}, \Delta G_{\mathrm{H}}$ at the Mo site also exhibits a cell size dependence and converges to $0.77 \mathrm{eV}$, which is close to $0.74 \mathrm{eV}$ reported by Huang et al. ${ }^{38}$ Clearly, the Mo site is going to have an extremely small occupancy of adsorbed hydrogen at room temperature because of the much higher $\Delta G_{\mathrm{H}}$ compared to that at the S site.
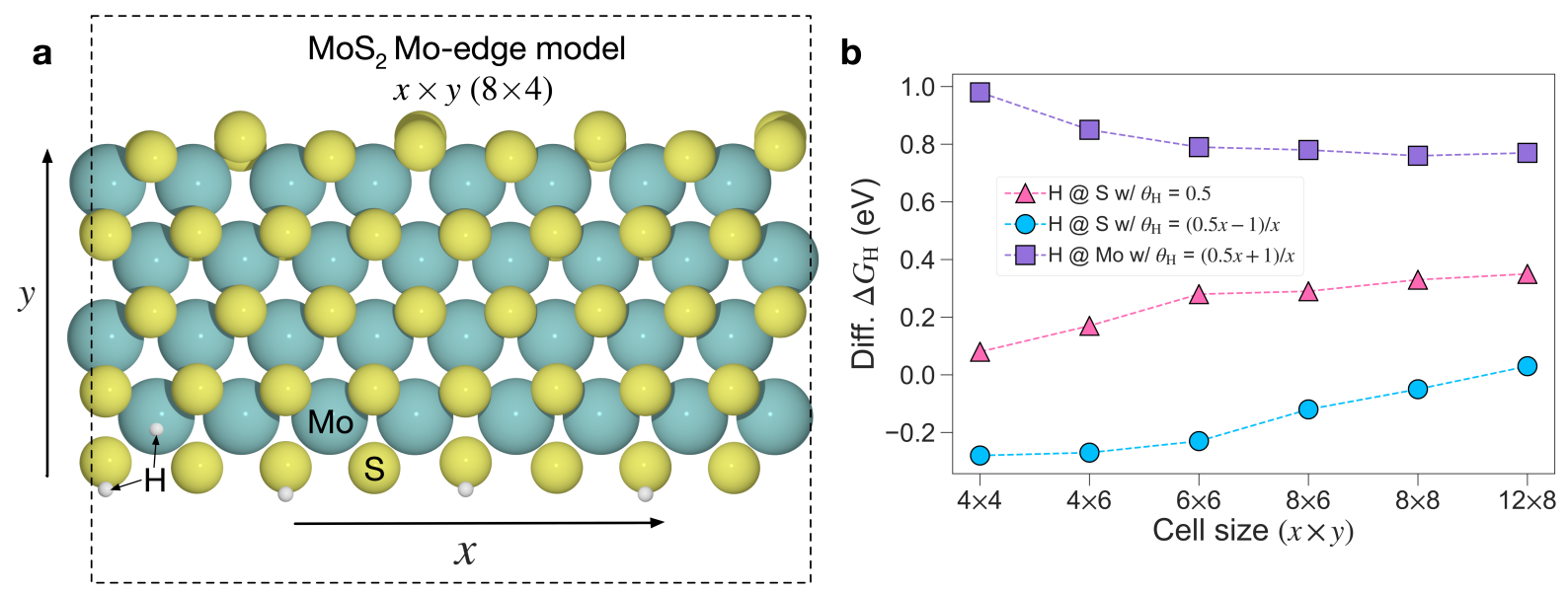

Figure 1. Calculated hydrogen adsorption free energy $\left(\Delta \mathbf{G}_{\mathbf{H}}\right)$. (a) Model of $\mathrm{MoS}_{2}$ with hydrogen coverage $\left(\theta_{\mathrm{H}}\right)$ of $0.5 \mathrm{ML}$ on the Mo-edge. The model size is described by $x \times y$, which represents the number of Mo atoms in the periodic unit cell in $\mathrm{x}$ - and $\mathrm{y}$ - directions. The Mo and $\mathrm{S}$ sites adsorbed with hydrogen are marked with arrows. (b) Calculated $\Delta \mathrm{G}_{\mathrm{H}}$ as a function of the cell size.

Heyrovsky reaction. Numerous experimental studies ${ }^{13-16,47}$ indicate that the hydrogen evolution occurs on $\mathrm{MoS}_{2}$ via the Volmer-Heyrovsky reaction, and previous computational work ${ }^{38,42,45}$ has shown that the Volmer barrier for proton transfer to the $\mathrm{S}$ ion at the $\mathrm{MoS}_{2}$ Mo-edge is very low. We will therefore focus on the second electron-proton transfer to adsorbed $\mathrm{H}$ to form $\mathrm{H}_{2}$ ( $a k a$. the Heyrovsky reaction). The Heyrovsky reaction was modelled with an explicit description of solvents determined on the surfaces using the constrained minima hopping algorithm ${ }^{33}$. 
Starting with a $4 \times 4$ cell, we found two reaction pathways, which are labeled as path-A and path-B in Figure $2 \mathrm{a}$ and $\mathrm{b}$, respectively. In both reaction paths, the adsorbed $\mathrm{H}$ at the $\mathrm{S}$ site reacts with a proton from waters to form $\mathrm{H}_{2}$. The difference between these two reaction paths lies in the transition state configurations. The transition state configuration of reaction path- $\mathrm{A}$ and path-B is $\mathrm{S}-\mathrm{H} \leftrightarrow \mathrm{H}-\mathrm{O}$ and $\mathrm{Mo}-\mathrm{H} \leftrightarrow \mathrm{H}-\mathrm{O}$, respectively. The calculated climbing-image nudged elastic band barrier of path-B is $0.41 \mathrm{eV}$ lower than that of path-A, suggesting the path-B would be dominant in the Heyrovsky reaction. Distinct from previous "H migration" mechanisms, ${ }^{38,45}$ we propose the Mo site only serves as a metastable transition state rather than a local potential minimum for adsorbed $\mathrm{H}$.
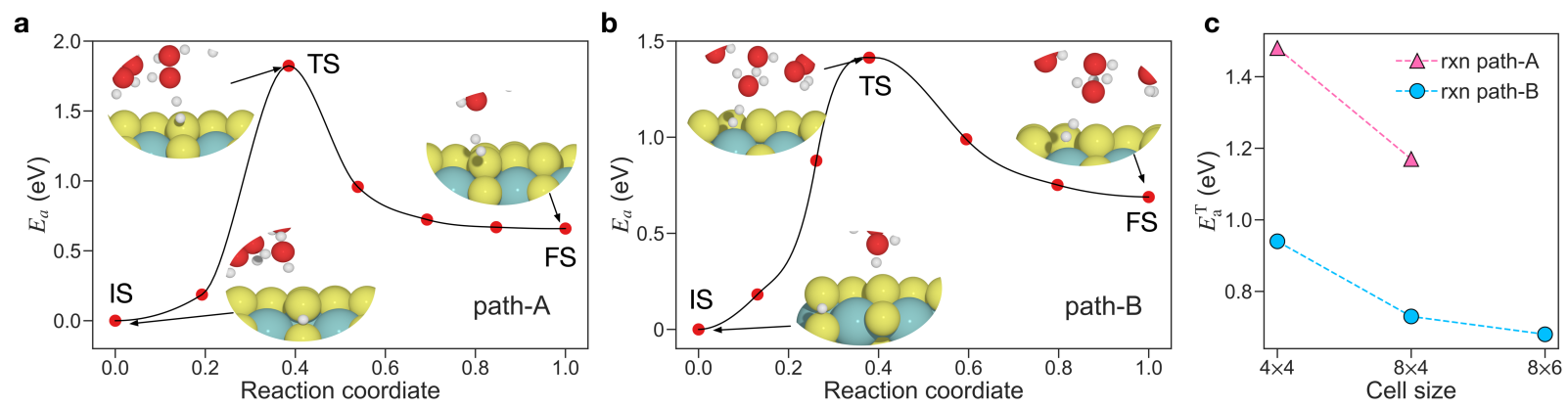

Figure 2. The Heyrovsky reaction. (a-b) Two minimum energy pathways of the Heyrovsky reaction on a $4 \times 4$ unit cell of $\mathrm{MoS}_{2}$. Insets show the side view of the initial state (IS), transition state (TS), and final state (FS) structures. (c) Charge-extrapolated Heyrovsky barriers as a function of the cell size at an electrode potential of 4.44 $\mathrm{V}$. The barriers for cell size convergence were all calculated with the hydrogen coverage of $0.5 \mathrm{ML}$ for consistency.

Similar to $\Delta G_{\mathrm{H}}$, we also examined the cell size effect on calculated barriers. Figure 2c presents the calculated $E_{a}^{T}$ with different cell sizes. Using an $8 \times 4$ cell, we also observe that the calculated $E_{a}^{T}$ of reaction path-B is $0.44 \mathrm{eV}$ lower than that of path-A, again verifying that the pathB would be dominant in the Heyrovsky reaction. Figure 2c clearly shows that the calculated $E_{a}^{T}$ of path-B decreases as the cell size increases. We ended the barrier calculations of path-B at the water/ $\mathrm{MoS}_{2}$ interface model by the $8 \times 6$ cell ( $\sim 220$ atoms). This is primarily because it is too computationally expensive to go beyond. The change of $E_{a}^{T}$ from $4 \times 4$ to $8 \times 4$ to $8 \times 6$ is $0.21 \mathrm{eV}$ 
and $0.05 \mathrm{eV}$, respectively. We therefore expect the energy difference in calculated $E_{a}^{T}$ between the $8 \times 4$ cell and the well-converged cell is small $(<0.1 \mathrm{eV})$.

The cell size dependence study of $\Delta G_{\mathrm{H}}$ reveals that the optimal $\theta_{\mathrm{H}}$ at the Mo-edge is 0.42 ML. Using an $8 \times 4$ cell, we obtained a calculated $E_{a}^{T}$ of $0.62 \mathrm{eV}$ at $\theta_{\mathrm{H}}$ of $0.38 \mathrm{ML}$, which is 0.11 $\mathrm{eV}$ smaller than that of $0.50 \mathrm{ML}$ (Figure 2c). We note that Tang and $\mathrm{Jiang}^{42}$ reported an activation energy of $0.62 \mathrm{eV}$ for the Heyrovsky reaction on the $1 \mathrm{~T} \mathrm{MoS} 2$ basal plane. These similar activation energies between $2 \mathrm{H} \mathrm{MoS}$ and $1 \mathrm{~T} \mathrm{MoS}$ verify the experimental finding ${ }^{17,51}$ that the catalytic activity between $2 \mathrm{H}$ and $1 \mathrm{~T}$ phases is not fundamentally different.

Scaling relationship and kinetic volcano. Next, we extended the calculations of the adsorption free energy and the activation barrier to include $\mathrm{MoSe}_{2}, \mathrm{WS}_{2}$, and $\mathrm{WSe}_{2}$. The results are summarized in Table 1. Except for $\mathrm{WSe}_{2}$, the calculated $\Delta G_{\mathrm{H}}$ of the rest are close to zero. The calculated $E_{a}^{T}$ of $\mathrm{MS}_{2}(\mathrm{M}=\mathrm{Mo} / \mathrm{W})$ is smaller than those of $\mathrm{MSe}_{2}(\mathrm{M}=\mathrm{Mo} / \mathrm{W})$. The data in Table 1 allow us to define a scaling relation between the Heyrovsky barrier $\left(E_{a}^{T}\right)$ and the hydrogen binding energy $\left(\Delta E_{\mathrm{H}}\right)$, as shown in Figure 3a. In the figure, we compare this relationship with the similar data for transition metals obtained from Tang et al. ${ }^{52}$. The two scaling relations show similar behavior with a constant up-shift in barriers of $\sim 0.4 \mathrm{eV}$ for the 2D-TMDs. This consistently higher activation barrier is therefore responsible for the lower absolute HER rates of 2D-TMDs.

Table 1. Calculated hydrogen adsorption free energies $\left(\Delta G_{\mathrm{H}}\right)$ and Heyrovsky barriers $\left(E_{a}^{T}\right)$ at electrode potential of $4.44 \mathrm{~V}$ for 2D-TMDs.

\begin{tabular}{ccc}
\hline Materials & $\Delta \boldsymbol{G}_{\mathbf{H}}(\mathbf{e V})$ & $\boldsymbol{E}_{\boldsymbol{a}}^{\boldsymbol{T}}(\mathbf{e V})$ \\
\hline $\mathrm{MoS}_{2}$ & 0.03 & 0.62 \\
$\mathrm{MoSe}_{2}$ & 0.09 & 0.74 \\
$\mathrm{WS}_{2}$ & -0.03 & 0.60
\end{tabular}




$\begin{array}{lll}\mathrm{WSe}_{2} & 0.24 & 0.84\end{array}$

Using the kinetic model and data in Figure 3a, we calculated the exchange current densities $\left(j_{0}\right)$ as a function of $\Delta G_{\mathrm{H}}$, shown in Figure $3 \mathrm{~b}$. The 2D-TMDs and the metals show two distinct volcanoes, separated by five to six orders of magnitude in rate. This difference is qualitatively consistent with what is observed in experiment (Figure 3c), where the difference is three to four orders of magnitude. ${ }^{7,13-15,46}$ The quantitative difference between theory and experiment amount to an error of $\sim 0.1 \mathrm{eV}$ in the calculated activation energy at room temperature for the two differentclasses of materials. This error is likely due to the limited cell size (Figure 2c) and/or accuracy of DFT calculations ${ }^{53,54}$. 
a

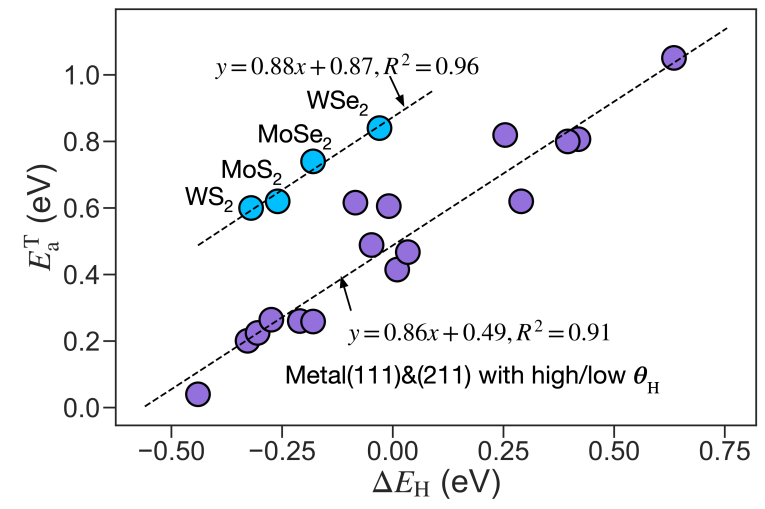

b

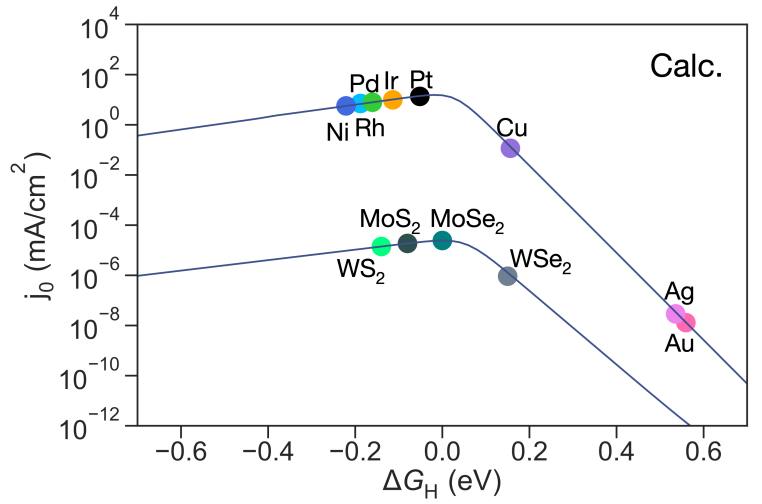

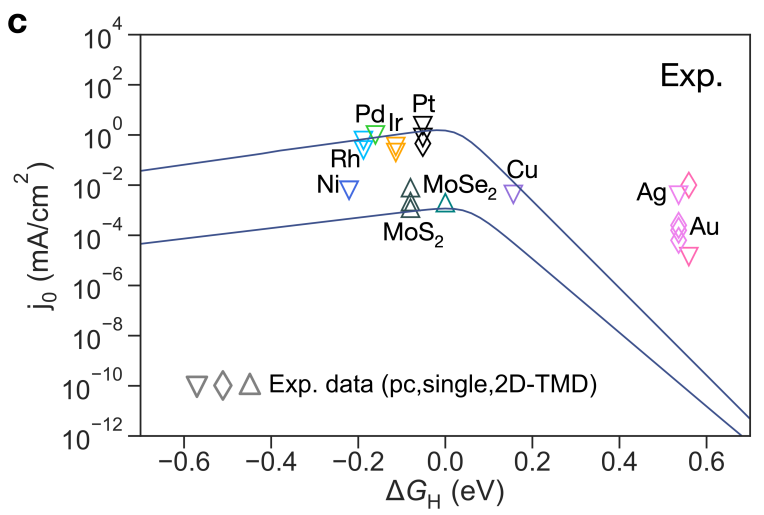

Figure 3. The HER activity of 2D-TMDs and metals. (a) Scaling relations of Heyrovsky barriers of 2D-TMDs and metals $v s$. hydrogen binding energy $\left(\Delta E_{\mathrm{H}}\right)$. (b) Calculated exchange current density $\left(j_{0}\right)$ as a function of $\Delta G_{\mathrm{H}}$. (c) Experimental exchange current density $\left(j_{0}\right)$ as a function of $\Delta G_{\mathrm{H}}$. The calculated and experimental $j_{0}$ of metals were taken from Ref 52 and 5,7,46, respectively. The experimental $j_{0}$ for 2D-TMDs were taken from Ref 13-15.

The existence of a scaling relation between $E_{a}^{T}$ and $\Delta E_{\mathrm{H}}$ for the 2D-TMDs suggests that $\Delta E_{\mathrm{H}}$, or, equivalently, the adsorption free energy, $\Delta G_{\mathrm{H}}$, is a good descriptor for the HER catalytic activity since both the stability of the intermediate and the activation energy are a function of $\Delta G_{\mathrm{H}}$, in contrast to the claims in Ref 38. Also, the shape and position of the maximum of the volcano plot for the two classes of materials match well with each other. This kinetic activity volcano further verifies that the thermodynamic activity descriptor, $\Delta G_{\mathrm{H}} \sim 0$ is a good design criterion for the 2D-TMDs as well as for the metals.

Origins of high activation barrier. On transition metals, the Heyrovsky step involves an association between a proton and a 'hydridic' hydrogen, which is slightly negatively charged. On 
2D-TMDs, on the other hand, the association is between a proton and a positively charged hydrogen bound to a chalcogenide ion. This gives an extra repulsion which is partially relieved by shifting the transition state to close to the transition metal which can transfer some electron charge to the receiving $\mathrm{H}$ atom. Figure 4 illustrates this by comparing charge density difference plots for $\mathrm{H}$ on $\mathrm{MoS}_{2}$ and $\mathrm{Pt}(111)$. The corresponding Bader charges are +0.04 e and -0.06 e, respectively. Similar results were also obtained for other catalysts. (Table S1) For a catalyst with $\Delta G_{\mathrm{H}} \sim 0$, if binding hydrogen with a positive charge, it might suffer from this higher activation energy. It is therefore an intrinsic property of this catalyst class.

a

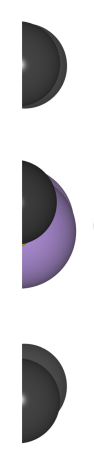

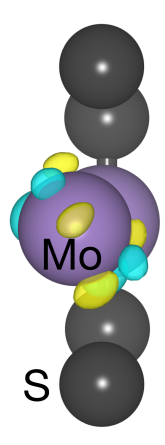
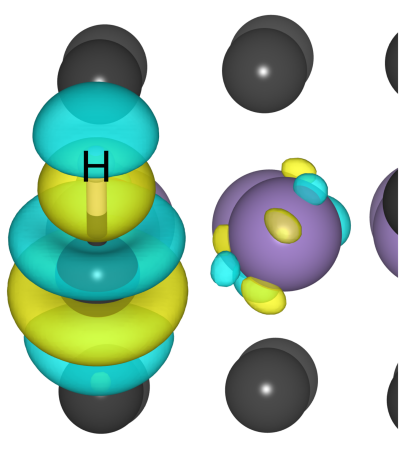

b

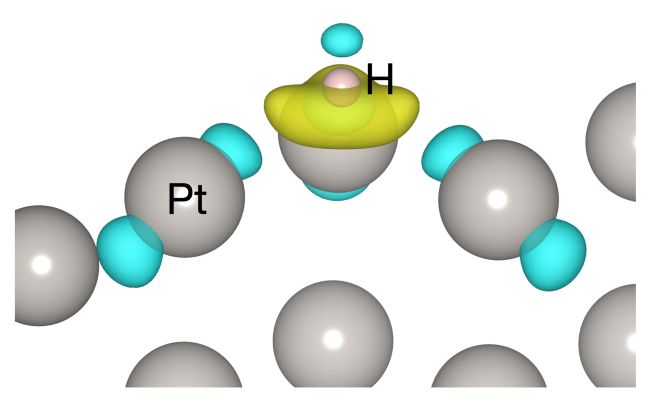

Figure 4. Charge density difference for $\mathrm{H}$ adsorbed on (a) $\mathrm{MoS}_{2}$ and (b) $\operatorname{Pt}(111)$. The yellow and cyan of density represent charge gain and loss, respectively. The charge density difference was drawn using VESTA ${ }^{54}$ with an equi-density level of $0.006 \mathrm{e} / a^{3}$, where $a_{0}$ is the Bohr radius.

\section{Discussion}

In general, the catalytic activity of a catalyst is determined by thermodynamics and kinetics. The existence of a scaling relation between the adsorption energy and the activation barrier establishes a link between these two factors. Some recent works ${ }^{33-38}$ show concerns about the effectiveness of the thermodynamic HER activity descriptor, $\Delta G_{\mathrm{H}}$ due to the missed kinetic contribution. Herein, based on the scaling relation and kinetic volcano of 2D-TMDs, we have verified that $\Delta G_{\mathrm{H}}$ still 
serves as a good descriptor of the HER activity for 2D-TMDs, and that $\Delta G_{\mathrm{H}} \sim 0$ remains a good criterion for optimal catalyst performance. Nevertheless, since 2D-TMDs have a different scaling relation from metals, one may have to go beyond 2D-TMDs or break this scaling relation to design a non-precious HER catalyst with the Pt activity. This is because the catalytic activity of 2D-TMDs is likely to be intrinsically limited by the scaling relation with relatively higher activation energies. Our results also indicate that the scaling of transition states must be considered when dealing with different classes of materials.

Equipped with these insights, we propose possible strategies to develop non-precious HER catalysts with activity approaching to Pt group metals - the best HER catalysts. Clearly, $\Delta G_{\mathrm{H}} \sim 0$ should be satisfied first. Moreover, considering a different scaling relation may exist for different classes of materials for HER, one needs to calculate the electrochemical barrier of the ratedetermining step. Here we suggest a negatively charged hydrogen may serve as a proxy to roughly estimate this barrier. This can be efficiently accessed by performing a Bader charge analysis for the compound of interest. Another approach is to explore compounds with metal ions as the active site for HER. One such compound could be a material with intercalated or interstitial metals. This is because the active site of metal ions might have a scaling relation same as transition metal electrocatalysts. An exploration of such materials will be the subject of future work.

\section{Conclusion}

In summary, we have thoroughly investigated the HER mechanism over 2D-TMDs through the lens of thermodynamics and kinetics. By revisiting $\Delta G_{\mathrm{H}}$ and activation barriers of $\mathrm{MoS}_{2}$, we have not only found an important dependence of cell size on their calculated values, but also proposed 
a new atomistic mechanism for the Heyrovsky reaction. More importantly, we have clarified that the lower HER rates of 2D-TMDs originate from their relatively higher Heyrovsky activation energies along with a different scaling relation and kinetic activity volcano from metals. This work provides crucial insights into the HER mechanism over 2D-TMDs and enables the effective design of non-precious HER catalysts with Pt activity.

\section{Methods}

DFT calculation. Spin-polarized DFT calculations were performed using Vienna ab initio simulation package within the projected-augmented wave method. ${ }^{55,56}$ The RPBE functional ${ }^{53}$ was used for the description of the exchange-correlation interaction. The plane wave energy cutoff was $400 \mathrm{eV}$. The electronic total energy and forces were converged to within $10^{-5} \mathrm{eV}$ and $0.05 \mathrm{eV} / \mathrm{A}$, respectively. More stringent convergence criteria of an energy cutoff of $520 \mathrm{eV}$ or a force criterion of $0.02 \mathrm{eV} / \mathrm{A}$ yield small difference $(<0.05 \mathrm{eV})$ in calculated hydrogen adsorption energies and electrochemical barriers.

An infinite stripe model was used to simulate the $10 \overline{1} 0 \mathrm{Mo} / \mathrm{W}$-edge and $1010 \mathrm{~S} / \mathrm{Se}$-edge, where the $\mathrm{Mo} / \mathrm{W}$-edge and $\mathrm{S} / \mathrm{Se}$-edge were covered with $50 \%$ sulfur/selenium and $100 \%$ sulfur/selenium, respectively. Previous work ${ }^{29,50,57}$ showed that this structure was active and the most energetically favorable under HER conditions. A vacuum separation of $12 \AA$ was added in both $y$ - and $z$-directions to eliminate the periodic image interaction. The Brillouin zone was sampled based on the Monkhorst-Pack scheme with the $k$-point grid of $2 \times 1 \times 1$ for the $4 \times 4$ stripe model. For models larger than $4 \times 4$, the gamma point was used. All structures used in this work were shared via figshare. 
The solvent was modeled using explicit water clusters determined on the surface of stripe models using the global optimization algorithm of constrained minima hopping.${ }^{58}$ In this method, Hookean constraints were applied to preserve water identify and prevent water evaporation. Molecular dynamic simulation (MD) and local structure optimization were carried out with total number of 50 steps. The energy threshold for determining a new local minimum was $2.5 \mathrm{eV}$ and the temperature for MD was $2000 \mathrm{~K}$. The force convergence criterion of local structure optimization was $0.05 \mathrm{eV} / \mathrm{A}$. The identified water clusters were then reoptimized using DFT and the lowest energy one was finally selected. We also tested the sensitivity of different water clusters on the calculated activation barrier at zero applied potential and found the effect of water structure on the barrier is small $(\leq 0.06 \mathrm{eV})$. (Table S2) The reaction barrier was calculated using the climbing-image nudged elastic band ${ }^{59}$ and constrained minimization implemented in Atomistic Simulation Environment. ${ }^{60}$ Vibrational frequencies calculations were then carried out to verify the transition state that has a single imaginary frequency.

Hydrogen adsorption free energy. The differential hydrogen adsorption energy $\left(\Delta G_{\mathrm{H}}\right)$ on the metal edge of 2D-TMDs, denoted by $\mathrm{MX}_{2}, \mathrm{M}=\mathrm{Mo}, \mathrm{W}, \mathrm{X}=\mathrm{S}, \mathrm{Se}$ ), was calculated by

$$
\Delta E_{H}=E\left[\mathrm{M} X_{2}+\mathrm{nH}\right]-\mathrm{E}\left[\mathrm{M} X_{2}+(\mathrm{n}-1) \mathrm{H}\right]-\frac{1}{2} E\left[H_{2}\right]
$$

where $\mathrm{E}\left[\mathrm{MX}_{2}+\mathrm{nH}\right]$ and $\mathrm{E}\left[\mathrm{MX}_{2}+(\mathrm{n}-1) \mathrm{H}\right]$ refer to the total energy of $\mathrm{MX}_{2}$ with $\mathrm{n}$ and $\mathrm{n}-1$ adsorbed hydrogen atoms on the metal edge. $\mathrm{E}\left[\mathrm{H}_{2}\right]$ is the total energy of a gas phase $\mathrm{H}_{2}$ molecule in a $15 \AA$ cubic box. $\Delta G_{\mathrm{H}}$ was then evaluated by

$$
\Delta G_{H}=\Delta E_{H}+\Delta E_{Z P E}-T \Delta S
$$

where $\Delta E_{\mathrm{ZPE}}$ and $T \Delta S$ are the difference in zero-point energy and entropy between gas phase $\mathrm{H}_{2}$ and adsorbed $\mathrm{H}$ at standard conditions. The calculated $\Delta E_{\mathrm{ZPE}}-T \Delta S$ for $\mathrm{MS}_{2}(\mathrm{M}=\mathrm{Mo}, \mathrm{W})$ and 
$\operatorname{MSe}_{2}(\mathrm{M}=\mathrm{Mo}, \mathrm{W})$ are $0.29 \mathrm{eV}$ and $0.27 \mathrm{eV}$, respectively. In the activity volcano plot, an $0.1 \mathrm{eV}$ correction was applied to the $\Delta G_{\mathrm{H}}$ reflecting the gas-phase error for $\mathrm{H}_{2}$ using the BEEF-vdw functional. ${ }^{61,62}$ This correction was consistent with previously calculated metal data. ${ }^{52}$ and resulted in an $0.05 \mathrm{eV}$ shift in $\Delta G_{\mathrm{H}}$, which was within DFT errors ${ }^{53,54}$ and did not alter the our conclusions.

Electrochemical barrier calculation. The charge-extrapolation method ${ }^{40}$ was adopted to deduce the electrochemical barrier. All charge-extrapolated barriers were referenced to the initial state of aqueous protons and electrons in bulk solution. As illustrated in Figure S1, the barrier at constant potential $\left(E_{a}^{T}\right)$ was given by:

$$
E_{a}^{T}=E_{a}-\Delta E+\Delta G_{C H E}=E_{b}+\Delta G_{C H E}
$$

where, $E_{a}$ is the forward reaction barrier, given by the energy difference between initial state (IS) and transition state (TS), $\left(E_{a}=E_{T S}-E_{I S}\right) ; E_{b}$ is the backward reaction barrier, given by the energy difference between transition state and final state (FS), $\left(E_{b}=E_{T S}-E_{F S}\right) ; \Delta E$ refers to the reaction energy, given by the energy difference between initial and final states. $\Delta E=E_{F S}-E_{I S} ; \Delta G_{C H E}$ is the hydrogen adsorption free energy determined using the computational hydrogen electrode ${ }^{63}$. The absolute potential at the water/2D-TMDs interface was given by the calculated work function. The applied potential was then determined by referencing to the standard hydrogen electrode potential of $4.44 \mathrm{~V}^{64}$. Similar to previous studies of the water/metals interfaces, ${ }^{52,65}$ a net dipole of $0.25 \mathrm{eV}$ was determined for waters/2D-TMDs and then subtracted from the calculated work function of the interface when determining the barrier at zero applied potential.

Microkinetic modelling. The hydrogen evolution reaction typically involves three elementary steps given by:

$$
H^{+}+e^{-} \rightarrow H^{*} \quad \text { (Volmer) }
$$




$$
\begin{aligned}
& H^{+}+e^{-}+H^{*} \rightarrow H_{2} \quad \text { (Heyrovsky) } \\
& H^{*}+H^{*} \rightarrow H_{2} \quad \text { (Tafel) }
\end{aligned}
$$

Here only the Volmer and Heyrovsky steps were used for the kinetic modeling since many experimental and theoretical works ${ }^{13-15,38,42,45}$ have indicated that HER takes place via the VolmerHeyrovksy reaction over 2D-TMDs. The rate constant was calculated by

$$
k=\frac{k_{B} T}{h} \exp \left(-\frac{G_{a}}{k_{B} T}\right) \exp \left(-\frac{\beta \eta}{k_{B} T}\right)
$$

where, $G_{a}$ is the activation free energy; $k_{B}$ is the Boltzmann constant; $h$ is Planck's constant and $T$ is the reaction temperature. The calculated $G_{a}$ for $2 \mathrm{D}$-TMDs were provided in Table S3. $\beta$ is the symmetry factor and equals to $0.5 ; \eta$ is the applied potential. The current density was then obtained by converting the reaction rates using the equation ${ }^{66}$ :

$$
j=\rho * 2 e * T O F
$$

where, $\rho$ is the surface density of active sites, $2 e$ is number of electrons for $\mathrm{H}_{2}$ production and TOF is the turnover frequency of electrons. Microkinetic modelling was carried out using the CatMAP package. ${ }^{67}$

\section{Supporting Information}

Calculated hydrogen adsorption energies, Bader charges and Heyrovsky barriers; Heyrovsky barriers with different water clusters; Illustration of charge extrapolation scheme; Calculated activation free energies and vibrational frequencies.

Notes: The authors declare no competing financial interest. 


\section{Acknowledgement}

This work was supported by Toyota Research Institute.

\section{Reference:}

(1) Voiry, D.; Yang, J.; Chhowalla, M. Recent Strategies for Improving the Catalytic Activity of 2D TMD Nanosheets Toward the Hydrogen Evolution Reaction. Adv. Mater. 2016, 28 (29), 6197-6206. https://doi.org/10.1002/adma.201505597.

(2) Lu, Q.; Yu, Y.; Ma, Q.; Chen, B.; Zhang, H. 2D Transition-Metal-DichalcogenideNanosheet-Based Composites for Photocatalytic and Electrocatalytic Hydrogen Evolution Reactions. Adv. Mater. 2016, 28 (10), 1917-1933. https://doi.org/10.1002/adma.201503270.

(3) Lin, L.; Sherrell, P.; Liu, Y.; Lei, W.; Zhang, S.; Zhang, H.; Wallace, G. G.; Chen, J. Engineered 2D Transition Metal Dichalcogenides-A Vision of Viable Hydrogen Evolution Reaction Catalysis. Adv. Energy Mater. 2020, 10 (16), 1-24. https://doi.org/10.1002/aenm.201903870.

(4) Parsons, R. The Rate of Electrolytic Hydrogen Evolution and the Heat of Adsorption of Hydrogen. Trans. Faraday Soc. 1958, 54, 1053. https://doi.org/10.1039/tf9585401053.

(5) Trasatti, S. Work Function, Electronegativity, and Electrochemical Behaviour of Metals. III. Electrolytic Hydrogen Evolution in Acid Solutions. J. Electroanal. Chem. 1972, 39 (1), 163-184. https://doi.org/10.1016/S0022-0728(72)80485-6.

(6) Barclay, D. J. The Possible Hydridic Nature of Adsorbed Hydrogen in the Hydrogen Evolution Reaction. J. Electroanal. Chem. Interfacial Electrochem. 1973, 44 (1), 47-51. https://doi.org/10.1016/S0022-0728(73)80513-3.

(7) Nørskov, J. K.; Bligaard, T.; Logadottir, A.; Kitchin, J. R.; Chen, J. G.; Pandelov, S.; Stimming, U. Trends in the Exchange Current for Hydrogen Evolution. J. Electrochem. Soc. 2005, 152 (3), J23. https://doi.org/10.1149/1.1856988.

(8) Kibler, L. A. Hydrogen Electrocatalysis. ChemPhysChem 2006, 7 (5), 985-991. https://doi.org/10.1002/cphc.200500646.

(9) Greeley, J.; Nørskov, J. K.; Kibler, L. A.; El-Aziz, A. M.; Kolb, D. M. Hydrogen Evolution Over Bimetallic Systems: Understanding the Trends. ChemPhysChem 2006, 7 (5), 10321035. https://doi.org/10.1002/cphc.200500663.

(10) Quaino, P.; Juarez, F.; Santos, E.; Schmickler, W. Volcano Plots in Hydrogen Electrocatalysis - Uses and Abuses. Beilstein J. Nanotechnol. 2014, 5 (1), 846-854. https://doi.org/10.3762/bjnano.5.96. 
(11) Lopato, E. M.; Eikey, E. A.; Simon, Z. C.; Back, S.; Tran, K.; Lewis, J.; Kowalewski, J. F.; Yazdi, S.; Kitchin, J. R.; Ulissi, Z. W.; Millstone, J. E.; Bernhard, S. Parallelized Screening of Characterized and DFT-Modeled Bimetallic Colloidal Cocatalysts for Photocatalytic Hydrogen Evolution. ACS Catal. 2020, $10 \quad$ (7), 4244-4252. https://doi.org/10.1021/acscatal.9b05404.

(12) Hinnemann, B.; Moses, P. G.; Bonde, J.; Jørgensen, K. P.; Nielsen, J. H.; Horch, S.; Chorkendorff, I.; Nørskov, J. K. Biomimetic Hydrogen Evolution: $\mathrm{MoS}_{2}$ Nanoparticles as Catalyst for Hydrogen Evolution. J. Am. Chem. Soc. 2005, 127 (15), 5308-5309. https://doi.org/10.1021/ja0504690.

(13) Jaramillo, T. F.; Jørgensen, K. P.; Bonde, J.; Nielsen, J. H.; Horch, S.; Chorkendorff, I. Identification of Active Edge Sites for Electrochemical $\mathrm{H}_{2}$ Evolution from MoS2 $\begin{array}{lllll}\text { Nanocatalysts. } & \text { Science } & \text { (80). } & \text { 2007, } & 317 \quad \text { (5834), }\end{array}$ https://doi.org/10.1126/science.1141483.

(14) Bonde, J.; Moses, P. G.; Jaramillo, T. F.; Nørskov, J. K.; Chorkendorff, I. Hydrogen Evolution on Nano-Particulate Transition Metal Sulfides. Faraday Discuss. 2008, 140, 219-231. https://doi.org/10.1039/b803857k.

(15) Kong, D.; Wang, H.; Cha, J. J.; Pasta, M.; Koski, K. J.; Yao, J.; Cui, Y. Synthesis of MoS 2 and $\mathrm{MoSe}_{2}$ Films with Vertically Aligned Layers. Nano Lett. 2013, 13 (3), 1341-1347. https://doi.org/10.1021/n1400258t.

(16) Ambrosi, A.; Sofer, Z; Pumera, M. 2H $\rightarrow$ 1T Phase Transition and Hydrogen Evolution Activity of $\mathrm{MoS}_{2}, \mathrm{MoSe}_{2}, \mathrm{WS}_{2}$ and $\mathrm{WSe}_{2}$ Strongly Depends on the $\mathrm{MX}_{2}$ Composition. Chem. Commun. 2015, 51 (40), 8450-8453. https://doi.org/10.1039/c5cc00803d.

(17) Yin, Y.; Han, J.; Zhang, Y.; Zhang, X.; Xu, P.; Yuan, Q.; Samad, L.; Wang, X.; Wang, Y.; Zhang, Z.; Zhang, P.; Cao, X.; Song, B.; Jin, S. Contributions of Phase, Sulfur Vacancies, and Edges to the Hydrogen Evolution Reaction Catalytic Activity of Porous Molybdenum Disulfide Nanosheets. J. Am. Chem. Soc. 2016, 138 (25), 7965-7972. https://doi.org/10.1021/jacs.6b03714.

(18) Yin, Y.; Zhang, Y.; Gao, T.; Yao, T.; Zhang, X.; Han, J.; Wang, X.; Zhang, Z.; Xu, P.; Zhang, P.; Cao, X.; Song, B.; Jin, S. Synergistic Phase and Disorder Engineering in 1T$\mathrm{MoSe}_{2}$ Nanosheets for Enhanced Hydrogen-Evolution Reaction. Adv. Mater. 2017, 29 (28), 1700311. https://doi.org/10.1002/adma.201700311.

(19) Toh, R. J.; Sofer, Z.; Luxa, J.; Sedmidubský, D.; Pumera, M. 3R Phase of $\mathrm{MoS}_{2}$ and $\mathrm{WS}_{2}$ Outperforms the Corresponding $2 \mathrm{H}$ Phase for Hydrogen Evolution. Chem. Commun. 2017, 53 (21), 3054-3057. https://doi.org/10.1039/c6cc09952a.

(20) Wu, Z.; Fang, B.; Bonakdarpour, A.; Sun, A.; Wilkinson, D. P.; Wang, D. WS 2 Nanosheets as a Highly Efficient Electrocatalyst for Hydrogen Evolution Reaction. Appl. Catal. B Environ. 2012, 125, 59-66. https://doi.org/10.1016/j.apcatb.2012.05.013.

(21) Cheng, L.; Huang, W.; Gong, Q.; Liu, C.; Liu, Z.; Li, Y.; Dai, H. Ultrathin $\mathrm{WS}_{2}$ Nanoflakes as a High-Performance Electrocatalyst for the Hydrogen Evolution Reaction. Angew. Chemie Int. Ed. 2014, 53 (30), 7860-7863. https://doi.org/10.1002/anie.201402315.

(22) Li, J.; Liu, P.; Qu, Y.; Liao, T.; Xiang, B. WSe 2 /RGO Hybrid Structure: A Stable and Efficient Catalyst for Hydrogen Evolution Reaction. Int. J. Hydrogen Energy 2018, 43 (5), 
2601-2609. https://doi.org/10.1016/j.ijhydene.2017.12.160.

(23) Wang, X.; Chen, Y.; Zheng, B.; Qi, F.; He, J.; Li, Q.; Li, P.; Zhang, W. Graphene-like $\mathrm{WSe}_{2}$ Nanosheets for Efficient and Stable Hydrogen Evolution. J. Alloys Compd. 2017, 691, 698704. https://doi.org/10.1016/j.jallcom.2016.08.305.

(24) Duan, J.; Chen, S.; Chambers, B. A.; Andersson, G. G.; Qiao, S. Z. 3D $\mathrm{WS}_{2}$ Nanolayers@Heteroatom-Doped Graphene Films as Hydrogen Evolution Catalyst Electrodes. $\quad A d v$. Mater. 2015, $27 \quad$ (28), 4234-4241. https://doi.org/10.1002/adma.201501692.

(25) Yang, J.; Mohmad, A. R.; Wang, Y.; Fullon, R.; Song, X.; Zhao, F.; Bozkurt, I.; Augustin, M.; Santos, E. J. G.; Shin, H. S.; Zhang, W.; Voiry, D.; Jeong, H. Y.; Chhowalla, M. Ultrahigh-Current-Density Niobium Disulfide Catalysts for Hydrogen Evolution. Nat. Mater. 2019, 18 (12), 1309-1314. https://doi.org/10.1038/s41563-019-0463-8.

(26) Saadi, F. H.; Carim, A. I.; Verlage, E.; Hemminger, J. C.; Lewis, N. S.; Soriaga, M. P. CoP as an Acid-Stable Active Electrocatalyst for the Hydrogen-Evolution Reaction: Electrochemical Synthesis, Interfacial Characterization and Performance Evaluation. $J$. Phys. Chem. C 2014, 118 (50), 29294-29300. https://doi.org/10.1021/jp5054452.

(27) Wan, C.; Regmi, Y. N.; Leonard, B. M. Multiple Phases of Molybdenum Carbide as Electrocatalysts for the Hydrogen Evolution Reaction. Angew. Chemie 2014, 126 (25), 6525-6528. https://doi.org/10.1002/ange.201402998.

(28) Tsai, C.; Abild-Pedersen, F.; Nørskov, J. K. Tuning the $\mathrm{MoS}_{2}$ Edge-Site Activity for Hydrogen Evolution via Support Interactions. Nano Lett. 2014, 14 (3), 1381-1387. https://doi.org/10.1021/nl404444k.

(29) Tsai, C.; Chan, K.; Abild-Pedersen, F.; Nørskov, J. K. Active Edge Sites in $\mathrm{MoSe}_{2}$ and $\mathrm{WSe}_{2}$ Catalysts for the Hydrogen Evolution Reaction: A Density Functional Study. Phys. Chem. Chem. Phys. 2014, 16 (26), 13156-13164. https://doi.org/10.1039/c4cp01237b.

(30) Tsai, C.; Chan, K.; Nørskov, J. K.; Abild-Pedersen, F. Theoretical Insights into the Hydrogen Evolution Activity of Layered Transition Metal Dichalcogenides. Surf. Sci. 2015, 640, 133-140. https://doi.org/10.1016/j.susc.2015.01.019.

(31) Er, D.; Ye, H.; Frey, N. C.; Kumar, H.; Lou, J.; Shenoy, V. B. Prediction of Enhanced Catalytic Activity for Hydrogen Evolution Reaction in Janus Transition Metal Dichalcogenides. Nano Lett. 2018, 18 (6), 3943-3949. https://doi.org/10.1021/acs.nanolett.8b01335.

(32) Noh, S. H.; Hwang, J.; Kang, J.; Seo, M. H.; Choi, D.; Han, B. Tuning the Catalytic Activity of Heterogeneous Two-Dimensional Transition Metal Dichalcogenides for Hydrogen Evolution. J. Mater. Chem. A 2018, 6 (41), 20005-20014. https://doi.org/10.1039/c8ta07141a.

(33) Lindgren, P.; Kastlunger, G.; Peterson, A. A. A Challenge to the $\mathrm{G} \sim 0$ Interpretation of Hydrogen Evolution. ACS Catal. 2020, 10 (1), 121-128. https://doi.org/10.1021/acscatal.9b02799.

(34) Koper, M. T. M. Analysis of Electrocatalytic Reaction Schemes: Distinction between RateDetermining and Potential-Determining Steps. J. Solid State Electrochem. 2013, 17 (2), 
339-344. https://doi.org/10.1007/s10008-012-1918-X.

(35) Ooka, H.; Nakamura, R. Shift of the Optimum Binding Energy at Higher Rates of Catalysis. J. Phys. Chem. Lett. 2019, 10 (21), 6706-6713. https://doi.org/10.1021/acs.jpclett.9b01796.

(36) Zeradjanin, A. R.; Grote, J.-P.; Polymeros, G.; Mayrhofer, K. J. J. A Critical Review on Hydrogen Evolution Electrocatalysis: Re-Exploring the Volcano-Relationship. Electroanalysis 2016, 28 (10), 2256-2269. https://doi.org/10.1002/elan.201600270.

(37) Exner, K. S. Does a Thermoneutral Electrocatalyst Correspond to the Apex of a Volcano Plot for a Simple Two-Electron Process? Angew. Chemie Int. Ed. 2020, 59 (26), 1023610240. https://doi.org/10.1002/anie.202003688.

(38) Huang, Y.; Nielsen, R. J.; Goddard, W. A.; Soriaga, M. P. The Reaction Mechanism with Free Energy Barriers for Electrochemical Dihydrogen Evolution on $\mathrm{MoS}_{2}$. J. Am. Chem. Soc. 2015, 137 (20), 6692-6698. https://doi.org/10.1021/jacs.5b03329.

(39) Schnur, S.; Groß, A. Challenges in the First-Principles Description of Reactions in Electrocatalysis. Catal. Today 2011, $165 \quad$ (1), 129-137. https://doi.org/10.1016/j.cattod.2010.11.071.

(40) Chan, K.; Nørskov, J. K. Potential Dependence of Electrochemical Barriers from Ab Initio Calculations. J. Phys. Chem. Lett. 2016, 7 (9), 1686-1690. https://doi.org/10.1021/acs.jpclett.6b00382.

(41) Groß, A. Structure of Electrode-Electrolyte Interfaces, Modeling of Double Layer and Electrode Potential. In Handbook of Materials Modeling; Andreoni, W., Yip, S., Eds.; Springer International Publishing: Cham, 2018; pp 1-34. https://doi.org/10.1007/978-3319-50257-1_7-1.

(42) Tang, Q.; Jiang, D. E. Mechanism of Hydrogen Evolution Reaction on 1T-MoS 2 from First Principles. ACS Catal. 2016, 6 (8), 4953-4961. https://doi.org/10.1021/acscatal.6b01211.

(43) Chen, S.; Chen, X.; Wang, G.; Liu, L.; He, Q.; Li, X. B.; Cui, N. Reaction Mechanism with Thermodynamic Structural Screening for Electrochemical Hydrogen Evolution on Monolayer 1T' Phase $\mathrm{MoS}_{2}$. Chem. Mater. 2018, 30 (15), 5404-5411. https://doi.org/10.1021/acs.chemmater.8b02236.

(44) He, Q.; Chen, X.; Chen, S.; Liu, L.; Zhou, F.; Li, X.-B.; Wang, G. Electrochemical Hydrogen Evolution at the Interface of Monolayer VS2 and Water from First-Principles Calculations. ACS Appl. Mater. Interfaces 2019, 11 (3), 2944-2949. https://doi.org/10.1021/acsami.8b17075.

(45) Ruffman, C.; Gordon, C. K.; Skúlason, E.; Garden, A. L. Mechanisms and PotentialDependent Energy Barriers for Hydrogen Evolution on Supported $\mathrm{MoS}_{2}$ Catalysts . J. Phys. Chem. C 2020. https://doi.org/10.1021/acs.jpcc.0c04146.

(46) Marković, N. M.; Grgur, B. N.; Ross, P. N. Temperature-Dependent Hydrogen Electrochemistry on Platinum Low-Index Single-Crystal Surfaces in Acid Solutions. $J$. Phys. Chem. B 1997, 101 (27), 5405-5413. https://doi.org/10.1021/jp970930d.

(47) Benck, J. D.; Hellstern, T. R.; Kibsgaard, J.; Chakthranont, P.; Jaramillo, T. F. Catalyzing the Hydrogen Evolution Reaction (HER) with Molybdenum Sulfide Nanomaterials. ACS Catal. 2014, 4 (11), 3957-3971. https://doi.org/10.1021/cs500923c. 
(48) Sheng, W.; Gasteiger, H. A.; Shao-Horn, Y. Hydrogen Oxidation and Evolution Reaction Kinetics on Platinum: Acid vs Alkaline Electrolytes. J. Electrochem. Soc. 2010, 157 (11), B1529. https://doi.org/10.1149/1.3483106.

(49) Hakala, M.; Kronberg, R.; Laasonen, K. Hydrogen Adsorption on Doped $\mathrm{MoS}_{2}$ Nanostructures. Sci. Rep. 2017, 7 (1), 1-13. https://doi.org/10.1038/s41598-017-15622-z.

(50) Rosen, A. S.; Notestein, J. M.; Snurr, R. Q. Comprehensive Phase Diagrams of $\mathrm{MoS}_{2}$ Edge Sites Using Dispersion-Corrected DFT Free Energy Calculations. J. Phys. Chem. C 2018, 122 (27), 15318-15329. https://doi.org/10.1021/acs.jpcc.8b02524.

(51) Voiry, D.; Salehi, M.; Silva, R.; Fujita, T.; Chen, M.; Asefa, T.; Shenoy, V. B.; Eda, G.; Chhowalla, M. Conducting $\mathrm{MoS}_{2}$ Nanosheets as Catalysts for Hydrogen Evolution Reaction. Nano Lett. 2013, 13 (12), 6222-6227. https://doi.org/10.1021/nl403661s.

(52) Tang, M. T.; Liu, X.; Ji, Y.; Norskov, J. K.; Chan, K. Modeling Hydrogen Evolution Reaction Kinetics through Explicit Water-Metal Interfaces. J. Phys. Chem. C 2020, 124 (51), 28083-28092. https://doi.org/10.1021/acs.jpcc.0c08310.

(53) Hammer, B.; Hansen, L. B.; Nørskov, J. K. Improved Adsorption Energetics within Density-Functional Theory Using Revised Perdew-Burke-Ernzerhof Functionals. Phys. Rev. B - Condens. Matter Mater. Phys. 1999, 59 (11), 7413-7421. https://doi.org/10.1103/PhysRevB.59.7413.

(54) Wellendorff, J.; Silbaugh, T. L.; Garcia-Pintos, D.; Nørskov, J. K.; Bligaard, T.; Studt, F.; Campbell, C. T. A Benchmark Database for Adsorption Bond Energies to Transition Metal Surfaces and Comparison to Selected DFT Functionals. Surf. Sci. 2015, 640, 36-44. https://doi.org/10.1016/j.susc.2015.03.023.

(55) Kresse, G.; Furthmüller, J. Efficient Iterative Schemes for Ab Initio Total-Energy Calculations Using a Plane-Wave Basis Set. Phys. Rev. B 1996, 54 (16), 11169-11186. https://doi.org/10.1103/PhysRevB.54.11169.

(56) Blöchl, P. E. Projector Augmented-Wave Method. Phys. Rev. B 1994, 50 (24), 1795317979. https://doi.org/10.1103/PhysRevB.50.17953.

(57) Prodhomme, P. Y.; Raybaud, P.; Toulhoat, H. Free-Energy Profiles along Reduction Pathways of MoS2 M-Edge and S-Edge by Dihydrogen: A First-Principles Study. J. Catal. 2011, 280 (2), 178-195. https://doi.org/10.1016/j.jcat.2011.03.017.

(58) Peterson, A. A. Global Optimization of Adsorbate-Surface Structures While Preserving Molecular Identity. Top. Catal. 2014, 57 (1-4), 40-53. https://doi.org/10.1007/s11244-0130161-8.

(59) Henkelman, G.; Jónsson, H. Improved Tangent Estimate in the Nudged Elastic Band Method for Finding Minimum Energy Paths and Saddle Points. J. Chem. Phys. 2000, 113 (22), 9978-9985. https://doi.org/10.1063/1.1323224.

(60) Hjorth Larsen, A.; JØrgen Mortensen, J.; Blomqvist, J.; Castelli, I. E.; Christensen, R.; Dułak, M.; Friis, J.; Groves, M. N.; Hammer, B.; Hargus, C.; Hermes, E. D.; Jennings, P. C.; Bjerre Jensen, P.; Kermode, J.; Kitchin, J. R.; Leonhard Kolsbjerg, E.; Kubal, J.; Kaasbjerg, K.; Lysgaard, S.; Bergmann Maronsson, J.; Maxson, T.; Olsen, T.; Pastewka, L.; Peterson, A.; Rostgaard, C.; SchiØtz, J.; Schütt, O.; Strange, M.; Thygesen, K. S.; 
Vegge, T.; Vilhelmsen, L.; Walter, M.; Zeng, Z.; Jacobsen, K. W. The Atomic Simulation Environment - A Python Library for Working with Atoms. J. Phys. Condens. Matter 2017, 29 (27). https://doi.org/10.1088/1361-648X/aa680e.

(61) Studt, F.; Behrens, M.; Kunkes, E. L.; Thomas, N.; Zander, S.; Tarasov, A.; Schumann, J.; Frei, E.; Varley, J. B.; Abild-Pedersen, F.; Nørskov, J. K.; Schlögl, R. The Mechanism of $\mathrm{CO}$ and $\mathrm{CO} 2$ Hydrogenation to Methanol over $\mathrm{Cu}$-Based Catalysts. ChemCatChem 2015, 7 (7), 1105-1111. https://doi.org/10.1002/cctc.201500123.

(62) Christensen, R.; Hansen, H. A.; Vegge, T. Identifying Systematic DFT Errors in Catalytic Reactions. Catal. Sci. Technol. 2015, $5 \quad$ (11), 4946-4949. https://doi.org/10.1039/c5cy01332a.

(63) Nørskov, J. K.; Rossmeisl, J.; Logadottir, A.; Lindqvist, L.; Kitchin, J. R.; Bligaard, T.; Jónsson, H. Origin of the Overpotential for Oxygen Reduction at a Fuel-Cell Cathode. $J$. Phys. Chem. B 2004, 108 (46), 17886-17892. https://doi.org/10.1021/jp047349j.

(64) Trasatti, S. The Absolute Electrode Potential: An Explanatory Note (Recommendations 1986). Pure Appl. Chem. 1986, 58 (7), 955-966. https://doi.org/10.1351/pac198658070955.

(65) Liu, X.; Xiao, J.; Peng, H.; Hong, X.; Chan, K.; Nørskov, J. K. Understanding Trends in Electrochemical Carbon Dioxide Reduction Rates. Nat. Commun. 2017, 8 (May), 1-7. https://doi.org/10.1038/ncomms15438.

(66) Hansen, H. A.; Viswanathan, V.; NØrskov, J. K. Unifying Kinetic and Thermodynamic Analysis of 2 E- and 4 e - Reduction of Oxygen on Metal Surfaces. J. Phys. Chem. C 2014, 118 (13), 6706-6718. https://doi.org/10.1021/jp4100608.

(67) Medford, A. J.; Shi, C.; Hoffmann, M. J.; Lausche, A. C.; Fitzgibbon, S. R.; Bligaard, T.; Nørskov, J. K. CatMAP: A Software Package for Descriptor-Based Microkinetic Mapping of Catalytic Trends. Catal. Letters 2015, 145 (3), 794-807. https://doi.org/10.1007/s10562015-1495-6.

TOC

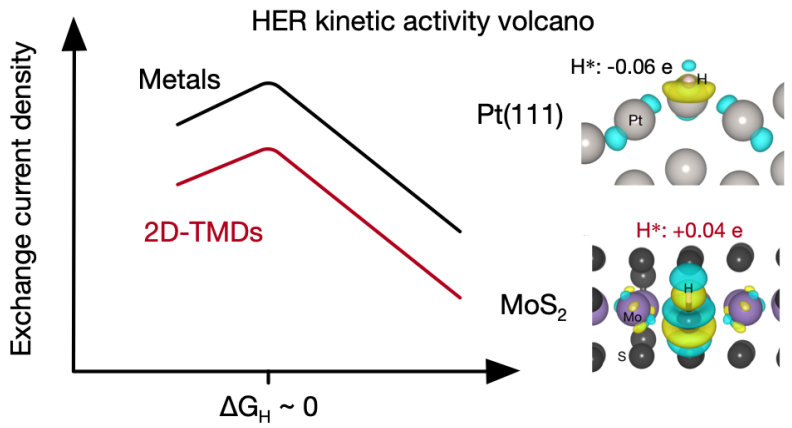

